\title{
Principales Obstáculos para la Transferencia de Conocimiento en los Centros e Institutos de Investigación del Triángulo del Café en Colombia
}

\author{
Carlos E. Marulanda(1), Francisco J. Valencia ${ }^{(2)}$ y Pablo F. Marín(1) \\ (1) Universidad Nacional de Colombia, Facultad de Administración, Departamento de Administración. Campus \\ Palogrande. Calle 27 No.64-60, Manizales-Colombia. (e-mail: cemarulandae@unal.edu.co; pfmarinc@nal.edu.co). \\ (2) Universidad Nacional de Colombia, Facultad de Administración, Departamento de Informática y Computación. Calle \\ 27 No.64-60, Manizales-Colombia. (e-mail: fjvalenciad@unal.edu.co).
}

Recibido Ago. 21, 2018; Aceptado Nov. 6, 2018; Versión final Nov. 30, 2018, Publicado Jun. 2019

\begin{abstract}
Resumen
El objetivo de este artículo es presentar los hallazgos relacionados con establecer los obstáculos que dificultan la transferencia de conocimiento de parte de los centros e institutos de investigación de la región triángulo del café de Colombia. Dicha valoración se realizó con un modelo de evaluación de transferencia de conocimiento, el cual se validó y aplicó a 24 centros e institutos de investigación de dicha región. Para el efecto se consideró un estudio de tipo descriptivo exploratorio y correlacional. Los resultados indican que los obstáculos para una efectiva transferencia de conocimiento se relacionan con la falta de una cultura organizacional que permita la transferencia, con el desconocimiento de convocatorias de proyectos, con los demasiados procesos organizacionales requeridos, y con la falta de uso de tecnologías de información.
\end{abstract}

\section{Main Obstacles for the Transfer of Knowledge in the Centers and Research Institutes of the Coffee Triangle in Colombia}

\begin{abstract}
The objective of this article is to present the findings related to establishing the obstacles that hinder the transfer of knowledge from the research centers and institutes of the coffee triangle region of Colombia. This assessment was carried out with a knowledge transfer evaluation model, which was validated and applied to 24 research centers and institutes in that region. For this purpose, an exploratory and correlative descriptive study was considered. The results indicate that the obstacles to an effective knowledge transfer are related with the lack of an organizational culture that allows the transfer, with the ignorance of calls for projects, with the too many required organizational processes, and with the lack of use of information technologies.
\end{abstract}

Keywords: knowledge transfer; knowledge management; research; projects 


\section{INTRODUCCIÓN}

El mundo empresarial y académico vienen estudiando y desarrollando en las últimas décadas investigaciones relacionadas con la gestión del conocimiento y más profundamente con la aplicación del concepto de transferencia de conocimiento, el cual se viene reconociendo como elemento diferenciador organizacional, es decir, la gestión se debe hacer realidad en la transferencia, y esto, en el caso de los centros e institutos de investigación, visibles en publicaciones, registro de software o patentes, entre otras. Esto como resultado de las tendencias que vienen siendo impulsadas por una economía mundial alineada a una mayor eficiencia, productividad y desarrollo tecnológico, que apunta a seguir generando mayores réditos de rentabilidad y más altas cifras de competitividad, para lo cual el trabajo colaborativo, el aprendizaje organizacional y las redes empresariales, entre otras, son fundamentales.

De acuerdo a esto, el papel de los centros e institutos de investigación, en este caso, del triángulo del café de Colombia (región centro occidente del País, conformada por los departamentos más productores del café colombiano, Caldas, Risaralda y Quindío y sus capitales Manizales, Pereira y Armenia), tienen una importancia preponderante, la cual nace desde el ejercicio de la investigación básica y aplicada, para la generación de nuevo conocimiento que puede convertirse en innovación de productos, procesos y servicios para el aparato productivo de ésta región del País. Centros e institutos de diversas áreas del conocimiento, pasando por el tema del café, la biotecnología, las ciencias de la salud, las ciencias jurídicas las ciencias sociales y humanas, las ciencias agrarias y la ingeniería entre otras. En este marco los resultados del que hacer investigativo debieran verse reflejados en publicaciones, registro de intangibles y patentes y para el efecto, se diseñó y valido un modelo de evaluación de transferencia de conocimiento, con el cual se hizo una valoración de los resultados del trabajo de éstos entes, conjugando aspectos fundamentales en el desarrollo organizacional, como es la cultura organizacional y los procesos organizacionales. Además, fue necesario identificar los diversos obstáculos que se presentan a diario en estas organizaciones que impiden un adecuado proceso de transferencia de conocimiento, los cuales se presentan en este artículo.

\section{Gestión de conocimiento (GC)}

Según (Al-Emran et al., 2018), el conocimiento es el activo más importante de cualquier organización; por lo tanto, tiene que ser gestionado de manera eficiente. La gestión del conocimiento (GC) se ha convertido en una predominante tendencia de investigación en el sector académico y empresarial. GC se define como el proceso de captura, almacenamiento, compartir y utilizar el conocimiento; es también un mecanismo emergente que puede encontrar Información particular más eficiente y organizarla para una rápida recuperación y reutilización. $\mathrm{La}(\mathrm{GC})$ se refiere a gestionar los procesos de una organización para aprovechar el conocimiento colectivo de la empresa, para lograr su sostenibilidad, mejorar la innovación y la capacidad de respuesta a los cambios del medio. La GC es reconocida como clave en las empresas y un proceso necesario para lograr ventaja competitiva dada la dificultad de imitación, desde la capacidad de la empresa para aplicar los conocimientos existentes y nuevos para crear nuevos productos y procesos (Santoro et al., 2018; Wingate, Smith, y Perk, 2018).

Explican (Colomo-Palacios et al., 2018), que la GC es vista como una de las piedras angulares para la calidad ya que comprende agregación, distribución, visualización de datos, e información y conocimiento para apoyar a los grupos de interés. Y es tan visible en la actualidad que según (Ardito et al., 2018), la Organización de Cooperación y Desarrollo Económicos (OCDE) y La Unión Europea (UE), han adoptado los marcos de GC en sus direcciones estratégicas para el desarrollo global y local. Ahora bien, (Mardani et al., 2018), establecen que los diferentes enfoques de GC se concentran en la creación, difusión, almacenamiento y aplicación de conocimientos para maximizar su efectividad; desarrollar una cultura intensiva en conocimiento; explorar y explotar el conocimiento; desarrollar el talento humano; desarrollo de procesos o facilitar la interacción e intercambio de conocimientos (Kambiz, Turkina, y Burger-Helmchen, 2018)

\section{Transferencia de conocimiento}

Schmidt et al. (2016), definen la transferencia de conocimiento como la transición efectiva de conocimiento de un actor a otro, que contiene dos aspectos: Además de la transición real del conocimiento, el conocimiento transferido tiene que llegar al destinatario de la manera correcta. Para hacer esto posible, la naturaleza del conocimiento y los requisitos previos de su transferencia deben ser conocidos. Para ser competitivo, vincular el conocimiento de las organizaciones involucradas es esencial. La logística de la transferencia de conocimiento describe la forma en que se transfiere el modo de conocimiento de un individuo o grupo u organización a otro(a) y se puede dividir en tres etapas: 1. Etapa de iniciación: el emisor debe decidir si quiere iniciar la transferencia, él tiene que elegir un destinatario adecuado, un canal, y qué conocimiento él quiere transferir. 2. Etapa de flujo de conocimiento: el conocimiento elegido fluye a través del canal prescrito. 3. 
Etapa de integración: esta etapa tiene lugar en el lado del destinatario: tiene que aprender sobre el conocimiento e integrarlo en su base de conocimiento.

Para la etapa de flujo de conocimiento, se usan varios métodos. La comunicación juega un papel fundamental en el proceso de la transferencia de conocimiento: sin él, sin flujo de conocimiento entre el remitente y el destinatario no es posible. La comunicación tiene que suceder en tres etapas: en el nivel de sintaxis, los datos son transferidos; en el nivel de la semántica, la información es transferida; en el nivel de la pragmática, el conocimiento es transferido. Para el caso de las empresas, la confianza es una de los atributos más importantes: tiene su origen en la actuación cooperativa y proporcionando información útil y exitosa relevante. Conocer a otros miembros del grupo de organizaciones o empresas sin contacto cara a cara hace que la construcción de la confianza sea más difícil o incluso imposible y dificulta la transferencia de conocimiento. En esta línea Bellini et al. (2016), explican que el conocimiento puede ser transferido entre individuos, equipos u organizaciones y se define como el proceso de aprendizaje a través de un intercambio interactivo de experiencias. La capacidad de acceder a la experiencia puede de hecho, garantizar la mejora continua en el tiempo y la creación de beneficios comerciales, por medio del intercambio de información y habilidades sistemáticamente organizadas.

Olmos-Peñuela et al., (2014), explican que existen las siguientes actividades de transferencia de conocimiento: 1) asesoramiento (servicios de asesoramiento técnico, encargado por organizaciones no académicas, que no involucran investigación académica original); 2) investigación por contrato (actividades originales de investigación llevadas a cabo por académicos y encargado por organizaciones no académicas); 3) investigación conjunta (actividades de investigación originales que implican arreglos de colaboración para realizar investigaciones emprendidas por académicos y organizaciones no académicas); 4) capacitación (actividades de aprendizaje, tales como cursos, ofrecidos por la comunidad académica (o demandada por no académicos) que están diseñados para necesidades de las organizaciones socioeconómicas (negocios, grupos gubernamentales y profesionales)); 5) movilidad de personal (flujo de académicos a otros entornos sociales (por ejemplo, adscripciones a empresas o al público organizaciones)). Igualmente, Azagra-Caroa et al., (2017), concluyen que algunos estudios muestran la importancia de la proximidad en los canales formales de transferencia, como la concesión de licencias y los contratos de investigación y desarrollo. El conocimiento se puede transferir a través de canales formales y locales, canales informales y no locales o combinaciones intermedias. La transferencia de conocimiento puede ser extremadamente valioso para mejorar las capacidades de los investigadores universitarios y centrar sus actividades en las necesidades de los usuarios.

A su vez Dinh y Mai (2015), concluyen que la transferencia de conocimiento se puede clasificar en cuatro categorías principales: (1) características firmes, (2) características universitarias, (3) relaciones entre empresas y universidades, y (4) canales de transferencia de conocimiento. El conocimiento puede ser explícito o implícito El conocimiento explícito está codificado y puede ser fácilmente transferido de entidades a entidades mientras que el conocimiento implícito tiene calidad personal que es difícil de transmitir. La transferencia de conocimiento es un concepto común, se puede entender como el intercambio de conocimiento entre los miembros participantes. Ocurre siempre que haya una fuente y un destinatario, es decir, la fuente y modelo de transferencia de conocimiento del receptor. $Y$ su éxito depende de las características de la fuente y el receptor, los cuales deben estar intrínsecamente motivados.

Complementan Aerts et al., (2017), explicando que la transferencia de conocimiento mejora la eficiencia que contribuye a la efectividad de las operaciones, por un lado, y a la innovación en términos de la calidad de la competencia, por otro lado. El objetivo de la transferencia es la creación de activos de conocimiento sin información y experiencia, y convirtiendo este conocimiento en una ventaja competitiva. Y su principal desafío reside en la instalación de dinámicas de aprendizaje organizacional que se adapten a la cultura de una organización y se basan en una combinación de personas (competencias) y sistemas de información (tecnología). La más importante herramienta de transferencia de conocimiento a nivel individual está en el entrenamiento, reportando a una documentación superior y personal, como una herramienta integral de transferencia que promueve el intercambio de información explícita, en todos los departamentos y dentro del organización, lo que permite el desarrollo de conocimiento organizacional. Finalmente (Bonfiglio et al., 2017), detallan que la transferencia de conocimiento es una condición previa para la innovación ya que crea alianzas y desarrolla capacidad. Por lo tanto, es un parte fundamental de los procesos y políticas de innovación. Por consiguiente, el foco está en las medidas que apoyan la transferencia de conocimiento e innovación.

\section{Modelo de transferencia de conocimiento}

Los resultados presentados, se apoyan en el modelo de transferencia de conocimiento para centros e institutos de investigación de (Marulanda et al., 2018), el cual se agrupa en 9 categorías: Dirección, cultura, aprendizaje, estándares, tecnologías, redes, servicios, producción comercial y producción intelectual; con sus 25 variables: direccionamiento, estructura, reglas PI, valores, clima, orientación, estrategia, competencias, 
roles, métodos, TI, organizaciones, investigación, contratos, alianzas, asesorías, consultorías, capacitaciones, patentes-licenciamiento, emprendimiento, artículos, libros, tesis, programas y ponencias. Desde un concepto de integralidad considerando el peso que tiene la transferencia al interior y al exterior de un centro e instituto de investigación.

\section{METODOLOGÍA}

La metodología se basó en el estudio desarrollado por Marulanda et. al, (2018), con base en los siguientes elementos: (i) delimitación de las unidades de observación; (ii) escala de medida; y (iii) categorías y variables evaluadas.

Delimitación de las unidades de observación: Básicamente las unidades de observación fueron los centros e institutos de investigación de la región triángulo del café de Colombia, de los cuales se logró integrar una población analizada de 24.

Escala de medida: Se formularon preguntas basadas en la escala de Likert, las cuales fueron calificadas de 1 a 5, donde la categorización es la siguiente: 1 , se está en desacuerdo o no realizado, 2 , realizado parcialmente, 3 , realizado en intervalos de tiempo, 4, realizado con regularidad y 5 realizado completamente. El grado de realización se refirió al impacto calificado de la variable respecto a la transferencia de conocimiento en las organizaciones.

Categorías y variables evaluadas: De acuerdo a las características de valoración, se agruparon las preguntas en las siguientes categorías y variables respecto a la cultura organizacional, como se detalla en la Tabla 1.

Basados en los planteamientos de Hair et al., (2007), frente a la evaluación de constructos teóricos, se considera el uso del análisis de correlación, el cual implica relacionar simultáneamente las variables establecidas entre sí, y resumir los resultados en una matriz de correlaciones.

Tabla1: Categorías y variables

\begin{tabular}{|l|l|}
\hline Categorías & Variables \\
\hline Redes & $\begin{array}{l}\text { Organizacionales (entre organizaciones y centros e institutos de investigación), de } \\
\text { investigación (entre centros, institutos, universidades e instituciones de educación } \\
\text { superior), contratos (con diversas organizaciones para el desarrollo de investigaciones) y } \\
\text { alianzas (con otras entidades, empresas u organismos para el desarrollo conjunto de } \\
\text { investigaciones) }\end{array}$ \\
\hline Servicios & $\begin{array}{l}\text { Asesoría (a entidades de diverso orden en lo relacionado con la experiencia adquirida en } \\
\text { la investigación de distintos temas de importancia), consultoría (con diversas } \\
\text { organizaciones para desarrollar conjuntamente planes, programas y yectos de } \\
\text { investigación) y capacitación (en diversos temas, procurando alineación con los resultados } \\
\text { últimos de investigación aplicada) }\end{array}$ \\
\hline Producción intelectual & $\begin{array}{l}\text { Artículos (en diversas revista nacionales e internacionales), libros (en papel o electrónicos, } \\
\text { de texto o de investigación, de diversas editoriales reconocidas) tesis (de maestría o } \\
\text { doctorado, producto de las investigaciones realizadas en los centros e institutos), creación } \\
\text { de programas (de pre o posgrado, producto del desarrollo de las investigaciones) y } \\
\text { ponencias (en diversos eventos de orden nacional o internacional). }\end{array}$ \\
\hline Obstáculos & $\begin{array}{l}\text { Desconocimiento (de convocatorias y otras), falta de financiación (recursos necesarios } \\
\text { para hacer visible la transferencia de conocimiento), tecnologías de información (dificultad } \\
\text { para incorporar el uso de recursos de TI), cultura (cultura organizacional para la } \\
\text { transferencia de conocimiento) y procesos (engorrosos o con una alta burocracia) }\end{array}$ \\
\hline
\end{tabular}

\section{RESULTADOS Y DISCUSIÓN}

Se presentan a continuación los hallazgos relacionados con la categoría redes, en los centros e institutos de investigación del triángulo del café de Colombia, que consideran las siguientes variables: Organización (organizac), de investigación (investiga), contratos y alianzas (complementando a (Marulanda et al., 2018)); en cuanto a la categoría de servicios, se consideraron las siguientes variables de medición: Asesorías, consultorías y capacitación y en cuanto a la categoría de producción intelectual, se consideraron las siguientes variables de medición: Artículos, libros, tesis, creación de programas de pre y posgrado (programas) y ponencias. Los resultados se pueden observar en la figura 1. 


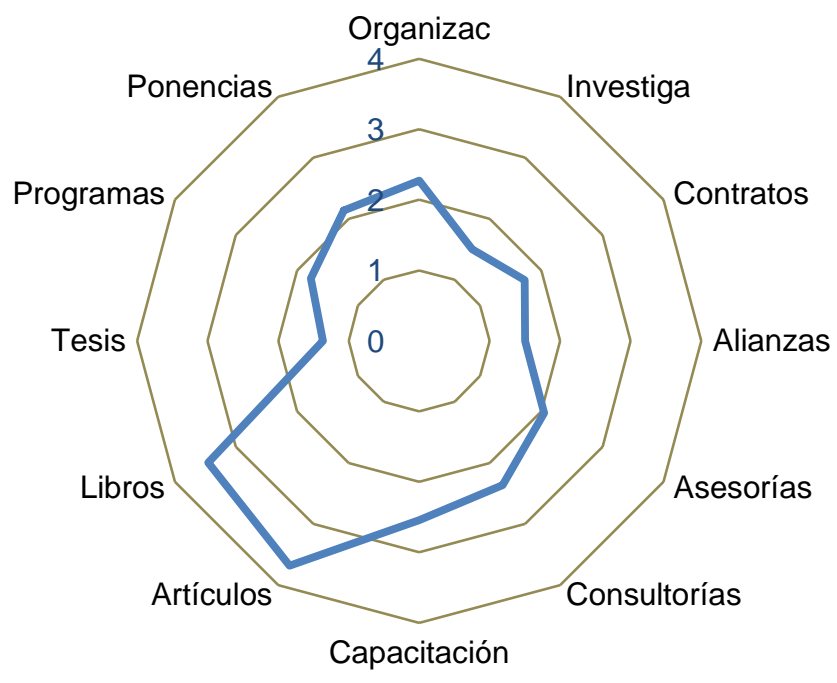

Fig. 1: Valoración de las categorías redes, servicios y producción intelectual

Los resultados de la valoración muestran que en términos de las redes que deben crearse o consolidarse en el marco de la transferencia de conocimiento, desde los centros e institutos hacia las organizaciones, entidades o empresas, se está por debajo de 3,0, sobre 5,0, alguna aproximación a 3,0 de parte de las redes organizacionales, pero las demás variables en 2,0 o por debajo de 2,0, lo que hace necesario fortalecer la posibilidad de crear y desarrollar mayores vínculos y más fuertes, con el fin de ofrecer posibilidades que respondan a las necesidades entre las partes. Los resultados de la valoración muestran que en términos de servicios que pueden ofertar los centros e institutos al medio, la valoración está por debajo de 3,0 sobre 5,0, una calificación muy baja, considerando las capacidades y recursos sobretodo en términos de capital intelectual que deben poseer dichos centros e institutos, quienes con los resultados de sus investigaciones deberían estar a la vanguardia en estos temas, tan importantes para el desarrollo y la ventaja competitiva de las compañías.

Los resultados de la valoración muestran que en términos de producción intelectual que pueden desarrollar los centros e institutos al medio, la valoración está por debajo de 3,0 sobre 5,0, una calificación muy baja, considerando que dichos centros e institutos en su mayoría son de universidades públicas y privadas de la región, las cuales contemplan de alguna manera en su misión, la creación de conocimiento. En este tema sí que hay por hacer, dadas las necesidades de las universidades de mantenerse en los altos puestos de producción científica del País. Ahora bien, considerando los resultados anteriores, se le indago a los centros e institutos sobre los obstáculos que se pudieran estar presentado para los resultados pobres que se están generando en su quehacer diario. Pare el efecto se evaluó la categoría obstáculos en los centros e institutos de investigación del triángulo del café de Colombia, y se consideraron las siguientes variables de medición: Desconocimiento (descono), falta de financiación (financ), tecnologías de información (TI), cultura (cultura) y procesos (proces). Los resultados se pueden observar en la figura 2.

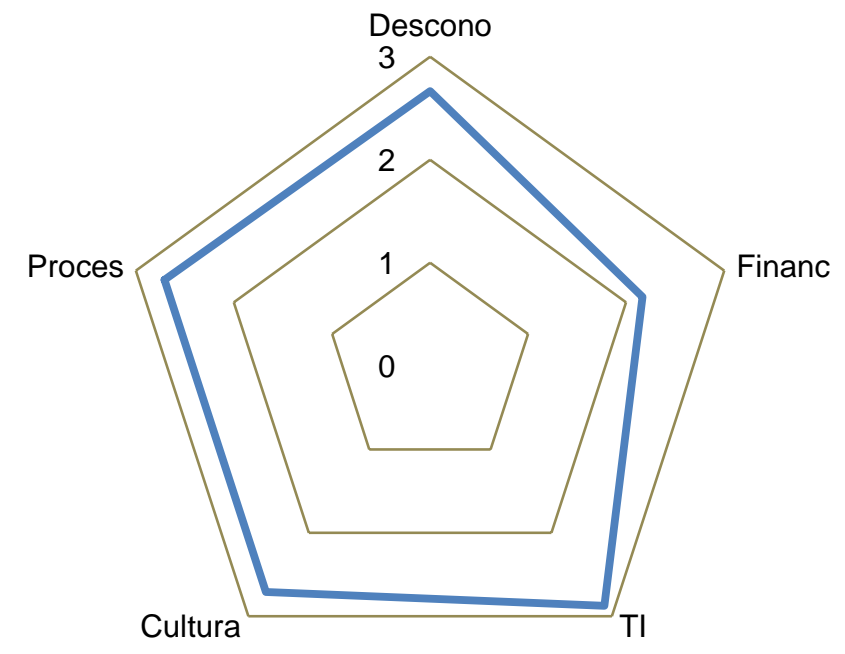

Fig. 2: Obstáculos para la transferencia de conocimiento 
Los resultados de la valoración muestran que en términos de obstáculos que pueden estar afectando el desarrollo de los centros e institutos de investigación, se califican casi que igual problemas de desconocimiento de convocatorias públicas o privadas para el desarrollo de investigaciones; los procesos que se desarrollan en los centros e institutos están dificultando su quehacer, producto de la burocracia excesiva; la cultura no está alineada con la misión y las TI, no se incorporan fácilmente por parte de los integrantes de estos centros e institutos. Se observa igualmente que no es tan problemático la falta de financiación. Considerando los bajos resultados de la transferencia se realizó un análisis de correlación, para determinar la asociación o relación entre las variables evaluadas y los resultados encontrados se pueden observar en la tabla 2.

Tabla 2: Matriz de correlación de variables

\begin{tabular}{|c|c|c|c|c|c|c|c|c|c|c|c|c|c|c|c|c|}
\hline & Organ & Invest & Contr & Alia & Ases & Cons & Arti & Lib & Tes & Prog & Pon & Desco & Apfin & $\mathrm{TI}$ & Cult & Proc \\
\hline Organ & 1 & & & & & & & & & & & & & & & \\
\hline Invest &, $548^{+*}$ & 1 & & & & & & & & & & & & & & \\
\hline Contr & ,256 & ,453* & 1 & & & & & & & & & & & & & \\
\hline Alia &, $415^{*}$ & ,497 &, $550^{* *}$ & 1 & & & & & & & & & & & & \\
\hline Ases &, $622^{* *}$ & 236 & ,352 & $441^{*}$ & 1 & & & & & & & & & & & \\
\hline Cons &, $456^{*}$ & 237 &, $559^{* *}$ & ,404 &, $764^{* *}$ & 1 & & & & & & & & & & \\
\hline Arti &,- 081 & ,358 & 079 & ,051 &,- 056 &,- 068 & 1 & & & & & & & & & \\
\hline Lib & -246 & -233 &,- 111 & 125 &,- 010 &,- 111 & ,350 & 1 & & & & & & & & \\
\hline Tes & -109 & ,098, & ,066 & 289 & ,071 & 106 & ,363 & 263 & 1 & & & & & & & \\
\hline Prog & -213 & -175 &,- 145 & -,175 &,- 139 &,- 250 &, $525^{* *}$ &, $414^{*}$ &, $416^{*}$ & 1 & & & & & & \\
\hline Pon & ,071 & 126 & -,046 & ,304 & ,020 &,- 020 &, $508^{*}$ &, $432^{*}$ & ,252 &, $438^{*}$ & 1 & & & & & \\
\hline Desco & -078 & -171 &,- 151 &,- 098 &,- 173 &,- 065 & -209 &, 000 &,- 062 & ,314 & ,069 & 1 & & & & \\
\hline Apfin &,- 043 & 255, & 270 & ,013 &,- 080 & ,214 &, $460^{*}$ & -184 &, $443^{*}$ & 293 & ,362 & -,031 & 1 & & & \\
\hline TI & -299 & -386 &,$- 478^{\star}$ & -386 &,$- 453^{*}$ &,- 190 & ,168 & 166 & ,144 & ,242 & 158 & 201 & 198 & 1 & & \\
\hline Cult & -302 &,- 049 & ,175 &,- 049 &,- 312 & ,103 & ,176 & 150 & ,103 & ,033 & ,040 & ,240 & ,363 & 342 & 1 & \\
\hline Proc & -347 & ,012 &,- 150 &,- 279 &,- 389 & -136 & ,166 & -091 &,- 021 &,- 081 & $\mid-, 210$ & ,023 & 306 & 195 &, $543^{* * *}$ & 1 \\
\hline
\end{tabular}

Los resultados de la correlación muestran que existe una relación directa y significativa entre las categorías y las variables valoradas, ya que los resultados expresan valores significativos por encima del $30 \%$ (en la tabla representado con ${ }^{* *}$ ). Ratifican que los resultados de la transferencia de conocimiento en los centros e institutos de investigación del triángulo del café de Colombia, están ligados a los diversos obstáculos presentados para el cumplimiento de su misión.

Estos resultados van en la misma línea de (Ali et al., 2012) y Wenpin (2001), quienes en su estudio concluyeron que la transferencia de conocimiento se da por las actividades de intercambio de conocimientos y por las dinámicas al interior y exterior de las organizaciones, las cuales se ven influenciadas por el intercambio interpersonal, es decir, las organizaciones comparten y se apoyan con el intercambio de conocimientos explícitos. También con lo encontrado por (Mehta et al., 2014), quienes evidencian que existen diversas problemáticas en la transferencia de conocimiento relacionadas con la cultura organizacional, la tecnología, los procesos, las actividades y otras variables que de una u otra manera retrasan o no permiten la posibilidad de intercambiar conocimientos ; En este sentido (Lee et al., 2016), concluyeron que la cultura organizacional es un factor de transferencia efectiva de conocimientos y las empresas deben identificar la cultura que apoya el conocimiento ya que a partir de conceptos como el clima organizacional, los valores, los principios, las costumbres, los hábitos y la forma de trabajo de las personas es que se puede llegar a intervenir efectivamente para lograr mayores niveles de transferencia de conocimiento. 
Igualmente, lo encontrado por (Sensuse et al., 2015) y Zappa y Robins (2016), quienes encontraron que la transferencia de conocimiento se afecta por el comportamiento individual y organizacional y tiene un impacto positivo o negativo en su implementación, lo que se explica desde el interés e iniciativa de los individuos para transferir conocimiento, pero en el marco de normas, procesos o guías organizacionales, reflejadas en el desarrollo y puesta en marcha de los incentivos, que pueden ser de orden monetario y social, y que apuntan a intervenir de una manera exitosa la posibilidad de transferencia de conocimiento de las personas en una organización. En este sentido, en los hallazgos de Chen, Hsiao, y Chu (2014), las actividades de replicación en la transferencia de conocimiento permiten a los empleados de las empresas trabajar juntos con sus socios en las empresas que envían y entienden el conocimiento que adquieren. A través de actividades de replicación las empresas podrán intensificar su competencia cooperativa con los socios. Además, la coordinación es posiblemente otro elemento crítico en el éxito transferencia de conocimiento entendiéndose como la medida en que las actividades, las personas, las rutinas, y las tareas trabajan juntas para lograr los objetivos generando más oportunidades para compartir conocimiento. En la transferencia de conocimiento, las actividades de replicación fortalecen las empresas, generan confianza en las relaciones con los socios, aumentan la comunicación en un lenguaje y símbolos compartidos y establecen consenso.

\section{CONCLUSIONES}

Del correspondiente análisis y discusión de los resultados, se desprenden las siguientes conclusiones:

(i) Los hallazgos presentados indican que hay deficiencias en la transferencia de conocimiento de los centros e institutos de investigación del triángulo del café de Colombia, los cuales vienen desde hace muchos años, generando esfuerzos importantes por vincularse mucho más con las necesidades y problemáticas del medio productivo y social de la región y el País. Para esto, el gobierno Nacional de Colombia, viene desarrollando una serie de estrategias que conduzcan a mayor transferencia e innovación de las empresas;

(ii) Se nota que hay problemas en el momento en que los centros e institutos de investigación puedan postularse a convocatorias públicas y privadas de proyectos de investigación, quizás por lo requisitos requeridos o por la falta de competencias para presentarse a las mismas;

(iii) Falta capacitación e inducción en el uso de herramientas de TI, como es el caso de uso de licencias de software especializado o de hardware de máximas prestaciones, para el desarrollo de los procesos de transferencia de conocimiento en los centros e institutos de investigación del triángulo del café;

(iv) Viene siendo un problema transversal en las entidades públicas de Colombia y se relaciona con la excesiva tramitología para firmar cualquier contrato, alianza o demás con un centro e instituto de investigación, dados los requisitos, tiempos, recursos y otros, lo que impide la participación de éstos;

v) Hay dificultades en una cultura organizacional que permita tener estructurados diversos programas que permitan adecuadamente la transferencia de conocimiento en los centros e institutos de investigación del triángulo del café

\section{AGRADECIMIENTOS}

A la Dirección de Investigaciones de la Universidad Nacional sede Manizales DIMA. Nombre de la convocatoria: Convocatoria nacional de proyectos para el fortalecimiento de la investigación, creación e innovación de la Universidad Nacional de Colombia. Proyecto de Investigación titulado: La transferencia de conocimiento en centros e institutos de investigación del triángulo del café. Código Hermes: 35721.

\section{REFERENCIAS}

Aerts, G., M. Dooms y E. Haezendonck, Knowledge transfers and project-based learning in large scale infrastructure development projects: an exploratory and comparative ex-post analysis, International Journal of Project Management, Issue 35, 224-240 (2017)

Al-Emran, M., V. Mezhuyev, A. Kamaludin y K. Shaalan, The impact of knowledge management processes on information systems: A systematic review, International Journal of Information Management, (43), 173-187 (2018)

Ali, N., D. Whiddett., A. Tretiakov e I. Hunter, The use of information technologies for knowledge sharing by secondary healthcare organisations in New Zealand, International journal of medical informatics, 8, 500-506 (2012) 
Ardito, L., A. Ferraris y otros tres autores, The role of universities in the knowledge management of smart city projects, Technological Forecasting and Social Change, 2-10 (2018)

Azagra-Caroa, J., D. Barberá-Tomása., M. Edwards-Schachtera y E. Tur, Dynamic interactions between universityindustry knowledge transfer channels: A case study of the most highly cited academic patent, Research Policy, 46, 463474 (2017)

Bellini, A., W. Aarseth, y A. Hosseini, Effective knowledge transfer in successful partnering projects, Energy Procedia, 96, 218-228 (2016)

Bonfiglio, A., B. Camaioni y otros tres autores, Are rural regions prioritizing knowledge transfer and innovation? Evidence from Rural Development Policy expenditure across the EU Space, Journal of Rural Studies, 53, 78-87 (2017)

Chen, C.-J., Y.-C. Hsiao y M.-A. Chu, Transfer mechanisms and knowledge transfer: The cooperative competency perspective, Journal of Business Research (67), 2531-2541 (2014)

Colomo-Palacios, R., E. Fernandes, P. Soto-Acosta y X. Larrucea, A case analysis of enabling continuous software deployment through knowledge management, International Journal of Information Management (40), 186-189 (2018)

Dinh, N. y N. Mai, Can knowledge be transferred from business schools to business organizations through in-service training students? SEM and fsQCA findings, Journal of Business Research, 68, 1332-1340 (2015)

Hair, J., R. Anderson, R. Tatham y W. Black, Análisis multivariante, Madrid, Pearson (2007)

Kambiz, S., E. Turkina y T. Burger-Helmchen, Knowledge management in OSS communities: Relationship between dense and sparse network structures, International Journal of Information Management, (38), 167-174 (2018)

Lee, J.-C., Y.-C. Shiue y C.-Y. Chen, Examining the impacts of organizational culture and top management support of knowledge sharing on the success of software process improvement. Computers in Human Behavior, 54, 462474 (2016)

Mardani, A., S. Nikoosokhan, M. Moradi, y M. Doustar, The Relationship Between Knowledge Management and Innovation Performance, Journal of High Technology Management Research, (29), 12-26 (2018)

Marulanda, C., O. Bedoya y H. Quintero, Modelo de transferencia de conocimiento para centros e institutos de investigación, Revista Espacios, (39), 1-20 (2018)

Marulanda, C., O. Bedoya y L. Martínez, Competencias personales para la transferencia de conocimiento en centros e institutos de investigación, Revista Espacios, (39), 28, 29-40 (2018)

Mehta, N., D. Hall y T. Byrd, Information technology and knowledge in software development teams: The role of project uncertainty, Information y Management, 51, 417-429 (2014)

Olmos-Peñuela, J., E. Castro-Martínez, y P. D’Este, Knowledge transfer activities in social sciences and humanities: Explaining the interactions of research groups with non-academic agents, Research Policy, 43, 696706 (2014)

Santoro, G., D. Vrontis, A. Thrassou y L. Dezi, The Internet of Things: Building a knowledge management system for open innovation and knowledge management capacity, Technological Forecasting \& Social Change (136), 347-354 (2018)

Schmidt, D., L. Böttcher y otros tres autores, Modeling Transfer of Knowledge in an Online Platform of a Cluster, Procedia CIRP, 50, 348-353 (2016)

Sensuse, D., E. Cahyaningsih y W. Wibowo, Knowledge Management: Organizational Culture in Indonesian Government Human Capital Management, Procedia Computer Science, 72, 485-494 (2015)

Wenpin, T., Knowledge Transfer in Intraorganizational Networks: Effects of Network Position and Absorptive Capacity on Business Unit Innovation and Performance, Academy of Management Journal, 44(5), 996-1004 (2001)

Wingate, L., N. Smith y E. Perk, The project vita: A dynamic knowledge management tool. Evaluation and Program Planning (71), 22-27 (2018)

Zappa, P. y G. Robins, Organizational learning across multi-level networks, Social Networks, 44, 295-306 (2016) 